\title{
Water-Stable, Nonsiliceous Hybrid Materials with Tunable Porosity and Functionality: Bridged Titania-Bisphosphonates
}

\author{
Yanhui Wang, Johan G. Alauzun,* and P. Hubert Mutin*
}

Cite This: Chem. Mater. 2020, 32, 2910-2918

Read Online

ABSTRACT: Combining the properties of organic and inorganic moieties with high surface areas and pore volumes offers endless possibilities to design materials adapted to a wide range of advanced applications. The vast majority of mesoporous hybrid materials are siliceous materials, and developing low-cost synthetic methodologies leading to water stable nonsiliceous hybrid materials with controlled texture and functionality is essential. We report here an original strategy for the synthesis of mesoporous bridged titaniabisphosphonate hybrids based on a one-step, templateless nonhydrolytic sol-gel route. The reaction of $\mathrm{Ti}\left(\mathrm{O}^{\mathrm{i}} \mathrm{Pr}\right)_{4}$ and a rigid bisphosphonate ester in the presence of $\mathrm{Ac}_{2} \mathrm{O}$ leads to the formation of $\mathrm{TiO}_{2}$ anatase nanorods cross-linked by fully condensed bisphosphonate groups. The porosity can be readily adjusted over a
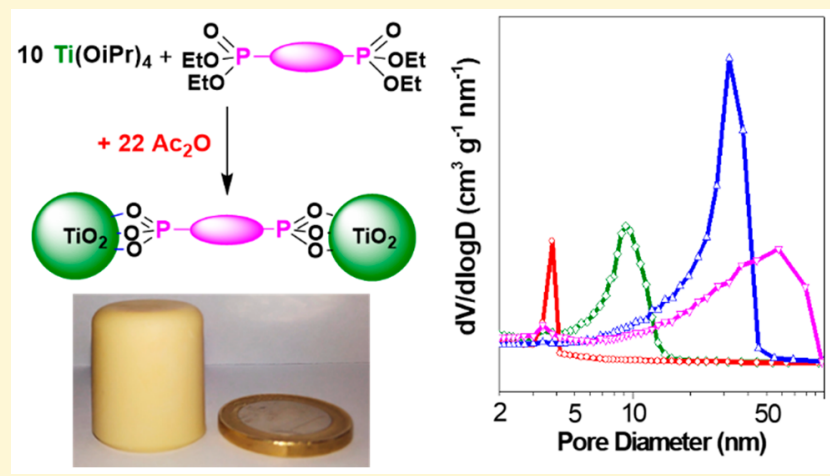
wide range by changing the reaction conditions, and very high specific surface areas (up to $720 \mathrm{~m}^{2} \mathrm{~g}^{-1}$ ) and pore volumes (up to $1.85 \mathrm{~cm}^{3} \mathrm{~g}^{-1}$ ) can be reached. The texture is stable in aqueous media between $\mathrm{pH} 1$ and $\mathrm{pH} 12$. Furthermore, accessible functional organic groups can be easily incorporated using either functional bisphosphonates or easily available monophosphonate compounds. The accessibility of bipyridyl organic groups was checked by $\mathrm{Cu}^{2+}$ adsorption from aqueous solutions. The unique combination of texture, functionality, and stability displayed by bridged titania-bisphosphonates makes these promising materials complementary of other hybrid materials such as organosilicas, MOFs, or mesoporous metal phosphonates.

\section{INTRODUCTION}

Over the last 30 years, mesoporous hybrid materials have received much attention because combining the properties of organic and inorganic moieties with high surface areas and pore volumes offers unprecedented possibilities to design materials adapted to a wide range of advanced applications. ${ }^{1,2}$

The vast majority of mesoporous hybrid materials with strong ionocovalent bonds between organic and inorganic species (Class II hybrid materials ${ }^{3}$ ) are siliceous materials based on organotrialkoxysilane coupling molecules. These compounds have been employed to prepare a large variety of hybrid materials with disordered or ordered mesoporosity, including surface modified silicas, (bridged) silsesquioxane gels and cogels, and periodic mesoporous organosilicas. ${ }^{4-8}$ Research efforts are increasingly shifting toward the development of nonsiliceous mesoporous hybrid materials. These materials are mostly based on carboxylate or phosphonate coupling molecules.

Carboxylate-based metal-organic frameworks (MOFs) have known an exponential growth during the past decade. ${ }^{9,10}$ They are renowned for their high crystallinity, extremely large surface areas, and well-defined micropores. However, designing mesoporous MOFs requires elaborated strategies, ${ }^{10,11}$ and the limited water stability of MOFs may be an issue. ${ }^{12}$
Phosphonates, which bind strongly to a wide range of metal atoms, are considered the best coupling molecules candidates for the design of water-stable, nonsiliceous class II hybrids. ${ }^{13-15}$ However, metal phosphonates classically yield dense layered structures with low intrinsic porosity. ${ }^{16}$

Several approaches have been used to obtain intrinsically porous metal phosphonates of titanium, zirconium, or aluminum, notably mixing bridging bisphosphonates and short monophosphonates ${ }^{13,17}$ to generate microporosity or using a bulky multiphosphonic acid to hinder the formation of layered structures. ${ }^{18}$

In addition, metal phosphonates with external mesoporosity have been prepared by sol-gel and/or hydrothermal processing using bis- or multiphosphonic acids. ${ }^{14,19}$ In these materials, the walls are built by amorphous or semicrystalline metal phosphonate particles with high phosphorus to metal ratios $(\mathrm{P} / \mathrm{M})$, ranging from approximately 1.3 to $2 .^{20-26} \mathrm{In}$

Received: December 10, 2019

Revised: $\quad$ March 21, 2020

Published: March 23, 2020 
Scheme 1. Schematic Representation of the Nonhydrolytic Sol-Gel Synthesis of bBzP-Ti and mBzP-Ti
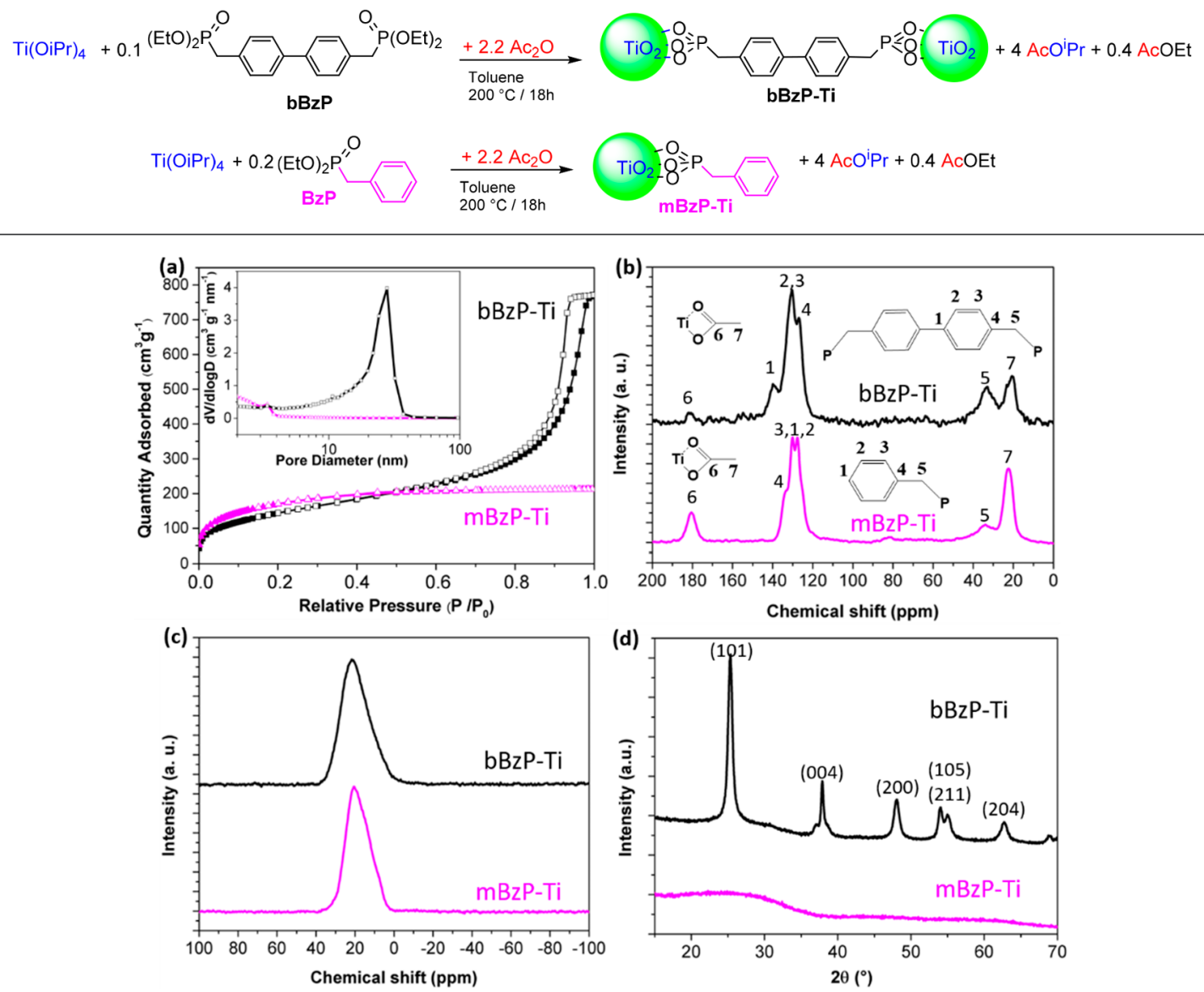

Figure 1. Comparison of the textural and structural characteristics of bBzP-Ti (black) and mBzP-Ti (magenta): (a) $\mathrm{N}_{2}$ adsorption-desorption isotherms at $77 \mathrm{~K}$ and corresponding $\mathrm{BJH}$ pore size distributions (inset); (b) ${ }^{13} \mathrm{C}$ solid-state CP-MAS NMR spectra; (c) ${ }^{31} \mathrm{P}$ solid-state MAS NMR spectra; (d) powder X-ray diffraction patterns. Its high specific surface area $\left(595 \mathrm{~m}^{2} \mathrm{~g}^{-1}\right)$ is located in micro- and mesopores smaller than $\sim 4 \mathrm{~nm}$.

most cases, the use of a template is required to reach specific surface areas and total pore volumes above $\sim 300 \mathrm{~m}^{2} \mathrm{~g}^{-1}$ and $\sim 0.30 \mathrm{~cm}^{3} \mathrm{~g}^{-1}$, respectively. ${ }^{14}$ For instance, an amorphous titanium phosphonate with hierarchical porosity and superior textural properties (surface area and pore volume of $448 \mathrm{~m}^{2}$ $\mathrm{g}^{-1}$ and $1.06 \mathrm{~cm}^{3} \mathrm{~g}^{-1}$, respectively) has been obtained through a hydrothermal process by reacting $\mathrm{TiCl}_{4}$ and 1-hydroxyethylidene-1,1-diphosphonic acid in the presence of both a cationic surfactant and an anionic polymeric template. ${ }^{27}$

However, the range of functional organic groups incorporated in metal phosphonate hybrid materials remains limited, and the cost of templates is a major drawback that hinder the industrial application of ordered mesoporous materials. Actually, the main advantages of periodic mesoporous materials lie in their high surface area and their large pore size, which minimizes diffusion limitations and improves accessibility for large molecules. For most applications, notably heterogeneous catalysis, the ordering of the porosity and even the narrow pore size distribution are not expected to bring any advantage, $^{28-31}$ and an aperiodic, three-dimensional mesoporous network (as in aerogels) can provide better accessibility and thus higher rates in applications involving mass-transfer. ${ }^{32}$

Accordingly, developing a template-free and general methodology for the synthesis of nonsiliceous hybrids with tunable mesoporosity and adjustable functionality remains highly desirable.
Metal oxide-phosphonates, built of metal oxide nanodomains modified by phosphonate groups, form another family of nonsiliceous hybrid materials. In this case, the $\mathrm{P} / \mathrm{M}$ ratio is much lower than in metal phosphonates $(0.02<\mathrm{P} / \mathrm{M}<$ $1)$. There have been very few attempts to prepare metal oxidephosphonates by sol-gel methods and up to now this approach has met with little success. ${ }^{33-35}$ Actually, the solgel synthesis of porous phosphonate-based hybrids is challenging, because of the very high reaction rates between precursors (phosphonic acids and metal alkoxides or chlorides). ${ }^{14,36}$

Nonhydrolytic sol-gel, which offers much more manageable rates, can provide an elegant solution to this problem. ${ }^{37}$ Recently, we used a nonhydrolytic route involving the reaction of titanium isopropoxide, acetophenone and diethyl octylphosphonate to prepare mesoporous titania-phosphonate hybrids, but the octylphosphonate groups prevented the growth of titania domains; as a result, the pore volume was moderate and decreased rapidly when increasing $\mathrm{P} / \mathrm{Ti}$ ratio. ${ }^{38}$

Here we show that the key to high pore volumes is to combine nonhydrolytic sol-gel with rigid bis-diethylphosphonate precursors. In this case, the growth of $\mathrm{TiO}_{2}$ is not prevented and the final material is built of anatase nanorods cross-linked by fully condensed bridging bisphosphonates. This very original structure leads, in the absence of a template, to outstanding textural properties (specific surface areas up to 830 

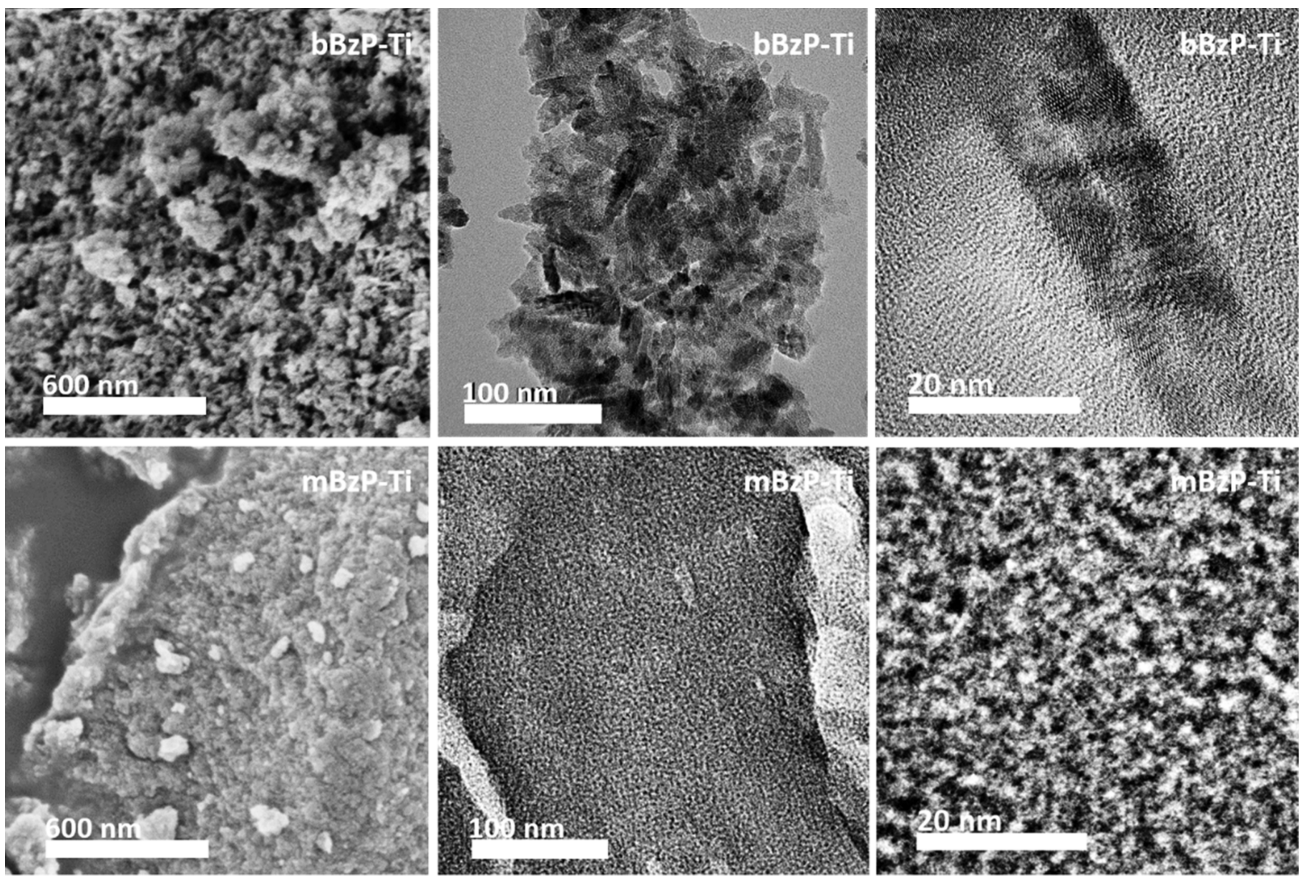

Figure 2. SEM (left) and TEM (middle, right) images of bBzP-Ti (top) and mBzP-Ti (bottom).

$\mathrm{m}^{2} \mathrm{~g}^{-1}$, pore volumes up to $1.85 \mathrm{~cm}^{3} \mathrm{~g}^{-1}$ ) and very high hydrolytic stability. Furthermore, the pore size can be easily tuned by changing the reaction conditions, and high amounts of accessible functional groups can be introduced using either functional bisphosphonate or monophosphonate groups. This low-cost method is general and can be extended to different bisphosphonate groups and metal precursors, giving access to a wide family of new mesoporous nonsiliceous hybrid materials.

\section{RESULTS AND DISCUSSION}

2.1. Synthesis and Characterization of bBzP-Ti. Recently, we reported that the reaction at $200{ }^{\circ} \mathrm{C}$ between $\mathrm{Ti}\left(\mathrm{O}^{\mathrm{i}} \mathrm{Pr}\right)_{4}$ and acetic anhydride afforded mesoporous anatase $\mathrm{TiO}_{2}$ with hierarchical porosity and high specific surface area. ${ }^{39}$ Here this nonhydrolytic route is extended to the synthesis of titania-bisphosphonates, using phosphonic acid diethyl esters as phosphonate precursors. Thus, a bridged titania bisphosphonate monolithic gel (denoted as bBzP-Ti) was prepared by reaction at $200{ }^{\circ} \mathrm{C}$ between $\mathrm{Ti}\left(\mathrm{O}^{\mathrm{i}} \mathrm{Pr}\right)_{4}$ and 0.1 equiv of $4,4^{\prime}$-bis(diethylphosphonomethyl)biphenyl in the presence of $\mathrm{Ac}_{2} \mathrm{O}$ (Scheme 1). The $\mathrm{P} / \mathrm{Ti}$ ratio is equal to 0.2 , which is 10 times lower than in a layered titanium phosphonate. For comparison, a sample with nonbridging groups $(\mathrm{mBzP}-\mathrm{Ti})$ was obtained under the same conditions but using 0.2 equiv of diethyl benzylphosphonate to keep the $\mathrm{P} / \mathrm{Ti}$ ratio constant. In both cases, the $\mathrm{P} / \mathrm{Ti}$ ratios measured by MEB-EDX are, within experimental error, identical to the expected ones, showing that all of the precursors are incorporated in the gel network and that the materials are homogeneous at the micrometer scale (see Table S1).

Nitrogen physisorption (Figure la) shows that bBzP-Ti is mainly mesoporous with a type $\operatorname{IV}(\mathrm{a})$ isotherm characteristic of mesoporous materials, ${ }^{40}$ a high specific surface area $\left(525 \mathrm{~m}^{2}\right.$ $\left.\mathrm{g}^{-1}\right)$ and an outstanding total pore volume $\left(1.20 \mathrm{~cm}^{3} \mathrm{~g}^{-1}\right)$. Such values are unprecedented for a nonsiliceous hybrid material prepared without a template. ${ }^{14}$ The pore size distribution confirms the presence of mesopores between 2 to $35 \mathrm{~nm}$, with a maximum at $25 \mathrm{~nm}$. The micropore volume determined by DFT method (Figure S1b) is quite low (0.08 $\left.\mathrm{cm}^{3} \mathrm{~g}^{-1}\right)$, comparable to the micropore volume found in SBA15 materials. $^{41}$ On the contrary, the nonbridged sample (mBzP-Ti) is mainly microporous, as shown by its type $\mathrm{I}(\mathrm{b})$ isotherm, with a low total pore volume $\left(0.33 \mathrm{~cm}^{3} \mathrm{~g}^{-1}\right)$ and a high micropore volume $\left(0.20 \mathrm{~cm}^{3} \mathrm{~g}^{-1}\right)$.

For comparison, a $\mathrm{TiO}_{2}$ sample prepared by the same nonhydrolytic route in toluene was purely mesoporous, with a specific surface area of $170 \mathrm{~m}^{2} \mathrm{~g}^{-1}$ and a pore volume of 0.36 $\mathrm{cm}^{3} \mathrm{~g}^{-1}$. ${ }^{39}$ Accordingly, the presence of phosphonate groups bridges favors the formation of a high specific surface area, but bisphosphonate bridges lead to a highly mesoporous material, whereas terminal phosphonates lead to a mostly microporous one.

In both cases, solid-state ${ }^{13} \mathrm{C}$ MAS NMR (Figure 1b) indicates that the bridging organic group was not damaged during the synthesis. Signals at 22 and $180 \mathrm{ppm}$ point to the presence of residual acetate groups, while the absence of resonances around $60 \mathrm{ppm}\left(\mathrm{O}_{\underline{C}} \mathrm{CH}_{2} \mathrm{CH}_{3}\right.$ sites $)$ and $75 \mathrm{pmm}$ $\left(\mathrm{O} \underline{\mathrm{CH}}\left(\mathrm{CH}_{3}\right)_{2}\right.$ sites $)$ indicates complete condensation of POEt and $\mathrm{Ti}\left(\mathrm{O}^{\mathrm{i}} \mathrm{Pr}\right)$ groups. The ${ }^{31} \mathrm{P}$ MAS NMR spectra (Figure 1c) display in both cases a single broad resonance centered at 20 ppm, ascribed to tridentate $\mathrm{C} \underline{\mathrm{P}}(\mathrm{OTi})_{3}$ sites. $^{33}$ FTIR spectroscopy (Figure S1a) confirms in both cases the presence of residual acetate groups (bands at 1450 and $1540 \mathrm{~cm}^{-1}$ ) and the predominantly tridentate bonding mode of phosphonate groups (absence of P-OC stretching vibration at $950 \mathrm{~cm}^{-1}$ and very low intensity of the $\mathrm{P}=\mathrm{O}$ stretching band at $\left.1250 \mathrm{~cm}^{-1}\right)$. The wide-angle powder XRD pattern of bBzP-Ti (Figure 1d) shows the presence of anisotropic $\mathrm{TiO}_{2}$ anatase nanoparticles elongated along the $c$-axis, as shown by the higher intensity and narrower (004) reflection compared to the (200) reflection. Such anatase nanorods have recently attracted attention as cathode materials for aluminum-ion batteries. ${ }^{42}$

The nanorods in bBzP-Ti are quite similar to those found for pure $\mathrm{TiO}_{2}$ samples prepared by the same nonhydrolytic 
route in toluene. ${ }^{39}$ The average crystallite size obtained by the Scherrer equation from the (101) and (200) peaks were 15 and $12 \mathrm{~nm}$, respectively. On the other hand, mBzP-Ti appears amorphous to $\mathrm{XRD}$, indicating that monophosphonate groups drastically hinder the growth of $\mathrm{TiO}_{2}$ domains, probably by capping the surface of these domains. In both cases, low angle XRD showed no evidence for a layered titanium (bis)phosphonate phase, which is the other stable phase in this system. As such a phase is usually non porous, its formation would be detrimental to the texture and to the organic groups accessibility. SEM and HR-TEM images (Figure 2 and Figure S2) confirm the presence in bBzP-Ti of anatase nanorods about $15 \mathrm{~nm}$ wide (in good agreement with the size determined by XRD) and up to $60 \mathrm{~nm}$ long. These nanorods are aggregated to form a three-dimensional porous network. On the other hand, mBzP-Ti appears built of compact aggregates of very small $(\sim 3 \mathrm{~nm})$ amorphous nanoparticles, with small pores $(<4 \mathrm{~nm})$. TEM-EDX images of bBzP-Ti (Figure S3) show that $\mathrm{P}$ atoms are homogeneously dispersed over the sample at the nanometer scale, confirming the absence of a separate titanium bisphosphonate phase.

The very different textures of bBzP-Ti and mBzP-Ti can be rationalized based on XRD and TEM results. Bisphosphonate groups do not hinder the formation of $\mathrm{TiO}_{2}$ nanorods, moreover the strong, covalent network formed by the nanorods linked by the fully condensed bisphosphonate groups prevents the collapse of pores during the drying step and accounts for the high mesopore volume.

On the contrary, terminal monophosphonate groups severely hinder the growth of the $\mathrm{TiO}_{2}$ domains and cannot link the resulting very small nanoparticles, which form compact, microporous aggregates.

2.2. Reactions Involved. In order to identify the reactions involved in this nonhydrolytic sol-gel route, we analyzed the reaction byproducts in the synthesis of bBzP-Ti by ${ }^{1} \mathrm{H}$ and ${ }^{13} \mathrm{C}$ liquid-state NMR (Figure S4). The only byproducts formed were isopropyl acetate and ethyl acetate $(>98 \%)$ in a 9:1 ratio. This is consistent with a clean, 2-step nonhydrolytic mechanism, involving first acetoxylation (eq 1) then condensation with ester elimination leading to the simultaneous formation of $\mathrm{Ti}-\mathrm{O}-\mathrm{Ti}$ bonds (eq 2) and $\mathrm{Ti}-\mathrm{O}-\mathrm{P}$ bonds (eq 3).

$$
\begin{aligned}
& \text { Ti-O } \mathrm{Pr}\left(\mathrm{CH}_{3} \mathrm{CO}\right)_{2} \mathrm{O} \rightarrow \mathrm{Ti}^{-O C O C H} \mathrm{CH}_{3} \mathrm{COO}^{\mathrm{i}} \mathrm{Pr} \\
& \mathrm{Ti}-\mathrm{O}{ }^{\mathrm{i}} \mathrm{Pr}+\mathrm{Ti}-\mathrm{OCOCH} \mathrm{CH}_{3} \rightarrow \mathrm{Ti}-\mathrm{O}-\mathrm{Ti}+\mathrm{CH}_{3} \mathrm{COO}^{\mathrm{i}} \mathrm{Pr} \\
& \mathrm{RP}-\mathrm{OEt}+\mathrm{Ti}-\mathrm{OCOCH}_{3} \rightarrow \mathrm{RP}-\mathrm{O}-\mathrm{Ti}+\mathrm{CH}_{3} \mathrm{COOEt}
\end{aligned}
$$

Therefore, the ideal reaction scheme for the synthesis of bBzP$\mathrm{Ti}$ is as depicted in eq 4.

$$
\begin{aligned}
& \mathrm{Ti}-\left(\mathrm{O}^{\mathrm{i}} \mathrm{Pr}\right)_{4}+0.1(\mathrm{EtO})_{2} \mathrm{PO}-\mathrm{bBz}-\mathrm{PO}(\mathrm{OEt})_{2}+2.2 \mathrm{Ac}_{2} \mathrm{O} \\
& \rightarrow \mathrm{Ti}(\mathrm{O})_{1.8}\left(\mathrm{O}_{3} \mathrm{P}-\mathrm{bBz}-\mathrm{PO}_{3}\right)_{0.1}+4 \mathrm{AcO}^{\mathrm{i}} \mathrm{Pr}+0.4 \mathrm{AcOEt}
\end{aligned}
$$

where $-\mathrm{bBz}$ - is the bridging biphenylenebis(methylene) group.

The amount of residual acetoxy groups can be evaluated from the thermogravimetric analysis in air of bBzP-Ti (Figure S5). The $22.5 \%$ weight loss between 120 and $600{ }^{\circ} \mathrm{C}$ would correspond to the conversion of $\mathrm{Ti}(\mathrm{O})_{1.68}(\mathrm{OAc})_{0.20}\left(\mathrm{O}_{3} \mathrm{P}-\mathrm{bBz}-\right.$ $\left.\mathrm{PO}_{3}\right)_{0.11}$ into $0.895 \mathrm{TiO}_{2}+0.105 \mathrm{TiP}_{2} \mathrm{O}_{7}$. The corresponding degree of condensation around $\mathrm{Ti}$ (given by $(1.68+2 \cdot 0.105)$ / $2 \cdot 100 \%)$ is very high, $94.5 \%$.
This nonhydrolytic route is particularly well-suited to the preparation of hybrid materials: the diethylphosphonate groups are compatible with most functional organic groups, and the byproducts formed are stable, volatile compounds, which facilitates their removal and avoids secondary reactions.

2.3. Shaping. Shaping porous materials is important for applications such as separation and catalysis. ${ }^{43-45}$ It is remarkable that in the case of bBzP-Ti a gentle drying at room temperature (without any solvent exchange) afforded a monolithic, crack-free xerogel (Figure 3). Again, this has to be
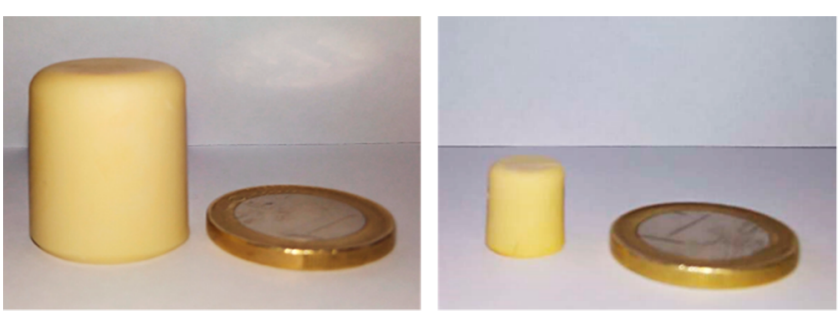

Figure 3. Monolithic bBzP-Ti gel (left) and bBzP-Ti xerogel (right) obtained by drying the gel at room temperature for $15 \mathrm{~h}$ under ambient pressure then for $2 \mathrm{~h}$ under vacuum.

ascribed to the strong, covalent network formed by the anatase nanorods linked by the fully condensed bisphosphonate groups, which prevents the formation of cracks during the evaporative drying.

2.4. Aqueous Stability of bBzP-Ti. In order to evaluate the aqueous stability of bBzP-Ti, the powder was stirred for 24 $\mathrm{h}$ at $30{ }^{\circ} \mathrm{C}$ in aqueous solutions at different $\mathrm{pH}$, washed, then dried at $120{ }^{\circ} \mathrm{C}$ under vacuum. As shown in Figure 4, for $\mathrm{pH}$

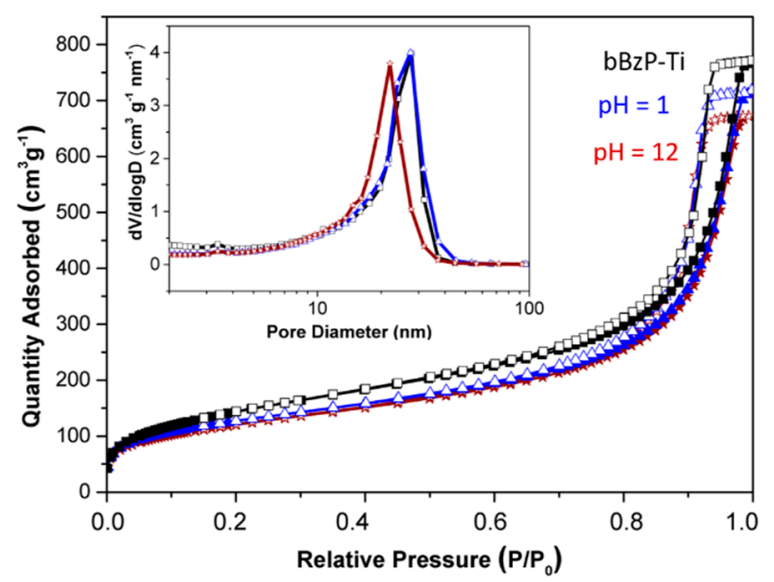

Figure 4. Evaluation of the water stability of bBzP-Ti under acidic and basic conditions: $\mathrm{N}_{2}$ physisorption isotherms at $77 \mathrm{~K}$ and corresponding $\mathrm{BJH}$ pore size distribution of bBzP-Ti (black), bBzPTi treated at $\mathrm{pH} 1$ (blue), and $\mathrm{pH} 12$ (brown) for $24 \mathrm{~h}$ at $30^{\circ} \mathrm{C}$. The filled and open symbols refer to adsorption and desorption, respectively.

values of 1 and 12 the physisorption isotherm and pore size distribution were only marginally impacted, and the specific surface area and total pore volume remained above $430 \mathrm{~m}^{2} \mathrm{~g}^{-1}$ and $1.0 \mathrm{~cm}^{3} \mathrm{~g}^{-1}$, respectively. Conversely, treatment at $\mathrm{pH} 0$ or $\mathrm{pH} 13$ reduced significantly the specific surface area and, in the case of $\mathrm{pH} \mathrm{13,} \mathrm{the} \mathrm{total} \mathrm{pore} \mathrm{volume.} \mathrm{In} \mathrm{addition,} \mathrm{the}$ mesoporous texture of $\mathrm{bBzP}-\mathrm{Ti}$ is maintained after treatment 


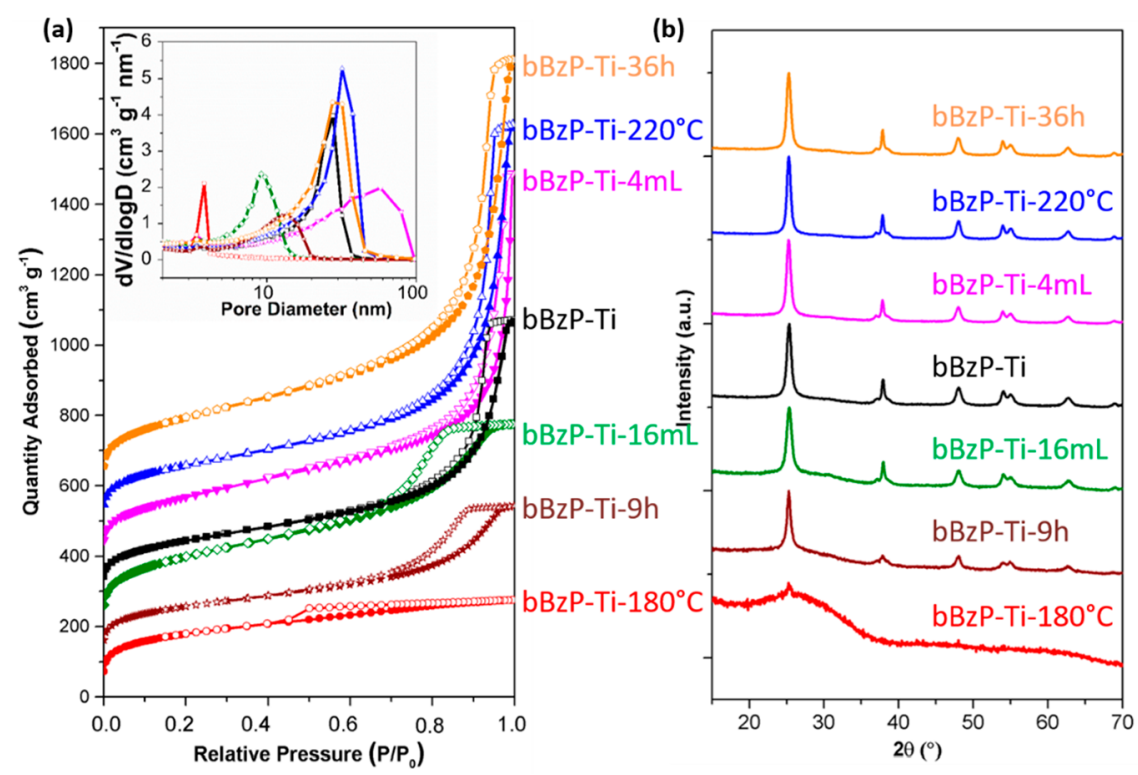

Figure 5. Characterization of bBzP-Ti samples prepared under different conditions: (a) $\mathrm{N}_{2}$ adsorption-desorption isotherm at $77 \mathrm{~K}$ and corresponding $\mathrm{BJH}$ pore size distribution (each isotherm curve, except the first one, was offset vertically by $100 \mathrm{~cm}^{3} \mathrm{~g}^{-1}$ ); $(\mathrm{b})$ powder X-ray diffraction patterns.

Table 1. Influence of Reaction Temperature $\left(T_{R}\right)$, Volume of Toluene $\left(V_{S}\right.$, for $\left.5.25 \mathrm{mmol} \operatorname{Ti}\left(\mathrm{O}^{\mathrm{i} P r}\right)_{4}\right)$, and Reaction Time $\left(t_{\mathrm{R}}\right)$ on the Texture of bBzP-Ti Samples

\begin{tabular}{|c|c|c|c|c|c|c|c|c|}
\hline sample & $T_{\mathrm{R}}\left({ }^{\circ} \mathrm{C}\right)$ & $V_{\mathrm{S}}(\mathrm{mL})$ & $t_{\mathrm{R}}(\mathrm{h})$ & $S_{\mathrm{BET}}{ }^{a}\left(\mathrm{~m}^{2} \mathrm{~g}^{-1}\right)$ & $V_{\mathrm{P}}^{b}\left(\mathrm{~cm}^{3} \mathrm{~g}^{-1}\right)$ & $V \mu^{c}\left(\mathrm{~cm}^{3} \mathrm{~g}^{-1}\right)$ & $D_{\mathrm{P}}^{d}(\mathrm{~nm})$ & $d_{\text {cryst }}^{e}(\mathrm{~nm})$ \\
\hline bBzP-Ti & 200 & 8 & 18 & 525 & 1.20 & 0.08 & 10.4 & 12 \\
\hline bBzP-Ti- $180{ }^{\circ} \mathrm{C}$ & 180 & 8 & 18 & 620 & 0.43 & 0.19 & 3.4 & am. \\
\hline bBzP-Ti-220 ${ }^{\circ} \mathrm{C}$ & 220 & 8 & 18 & 575 & 1.75 & 0.07 & 13.4 & 15 \\
\hline bBzP-Ti-4 mL & 200 & 4 & 18 & 625 & 1.69 & 0.05 & 18.2 & 13 \\
\hline bBzP-Ti-16 mL & 200 & 16 & 18 & 720 & 0.89 & 0.13 & 5.7 & 8 \\
\hline bBzP-Ti-9h & 200 & 8 & 9 & 570 & 0.73 & 0.15 & 7.0 & 9 \\
\hline bBzP-Ti-36h & 200 & 8 & 36 & 715 & 1.85 & $<0.01$ & 11.4 & 14 \\
\hline
\end{tabular}

${ }^{a}$ BET specific surface area. ${ }^{b}$ Total pore volume at $P / P_{0}=0.99 .{ }^{c}$ Volume of micropores estimated by DFT analysis. ${ }^{d} \mathrm{BJH}$ average pore diameter in the $2-100 \mathrm{~nm}$ range calculated from the desorption branch. ${ }^{e}$ Average crystallite size estimated by the Scherrer equation from the width of the (101) reflection.

in neutral water at $100{ }^{\circ} \mathrm{C}$ for $2 \mathrm{~h}$ (see Table S2 and Figure S6).

The remarkable water stability of bBzP-Ti can be explained by its structure: both $\mathrm{Ti}-\mathrm{O}-\mathrm{Ti}$ and $\mathrm{Ti}-\mathrm{O}-\mathrm{P}$ bonds are known for their high hydrolytic stability, as shown by the very low aqueous solubility of crystalline $\mathrm{TiO}_{2}{ }^{46}$ and of $\mathrm{M}(\mathrm{IV})$ phosphonates. $^{47}$

2.5. Tuning the Texture of bBzP-Ti. As demonstrated in Figure 5 and Figure S7, playing on the reactions kinetics offers simple and efficient ways to tune the texture of bridged titaniabisphosphonates, from micromesoporous to meso-macroporous. Starting from the "standard" synthesis of bBzP-Ti, performed with $8 \mathrm{~mL}$ of toluene (for $5.25 \mathrm{mmol} \mathrm{Ti}\left(\mathrm{O}^{\mathrm{i}} \mathrm{Pr}_{4}\right)$ ) at $200{ }^{\circ} \mathrm{C}$ for $18 \mathrm{~h}$, different bBzP-Ti samples were prepared by changing either the reaction temperature, the solvent volume, or the reaction time. Depending on these reaction conditions, the specific surface area varies between 525 and $720 \mathrm{~cm}^{2} \mathrm{~g}^{-1}$ and the pore volume from 0.43 to $1.85 \mathrm{~cm}^{3} \mathrm{~g}^{-1}$ (Table 1). As shown on the $\mathrm{BJH}$ pore size distributions (Figure 5), the maximum pore size varies from $100 \mathrm{~nm}$ to less than $5 \mathrm{~nm}$. In all cases, the ${ }^{31} \mathrm{P}$ MAS NMR spectra are similar, pointing to a complete condensation of phosphonate precursors. On the other hand, the crystallinity of the samples depends on the reaction conditions: the size of the anatase crystallites varies from 8 to $15 \mathrm{~nm}$, and the sample obtained at $180{ }^{\circ} \mathrm{C}$ is practically amorphous. As a rule, a higher reaction temperature, a higher concentration, or a longer reaction time lead to a higher mesopore volume, a lower micropore volume and larger micropores (Figure S8), and a higher crystallite size.

2.6. Introducing Organic Functionality. A major advantage of hybrid materials is the possibility to tailor the functional organic groups depending on the targeted applications. We explored several approaches for preparing bridged titania bisphosphonate materials with functional organic groups. The first approach consists in using functional bisphosphonate bridges. As the number of commercially available bridging bisphosphonates is limited, an interesting alternative consists in modifying a simple (nonfunctional) bridged bisphosphonate with functional monophosphonate groups, either by co-condensation or by postmodification (Scheme 2).

In order to demonstrate the first approach, we prepared a titania bisphosphonate sample with bridging bipyridyl groups (bPyP-Ti) by exactly the same synthesis as bBzP-Ti but using tetraethyl 2,2'-bipyridine-5,5'-bisphosphonate (bPyP) instead of bBzP. As shown in Figure 6, this functional material is mesoporous, with high specific surface area $\left(520 \mathrm{~m}^{2} \mathrm{~g}^{-1}\right)$ and pore volume $\left(0.93 \mathrm{~cm}^{3} \mathrm{~g}^{-1}\right)$ (Table 2). 
Scheme 2. Schematic Representation of the Different Functional Bridged Titania-Bisphosphonates Prepared: Using a Functional Bisphosphonate (bPyP-Ti), by Cogelation (bBzP-PyP-Ti), or by Surface Modification of bBzP-Ti (BPPA/bBzP-Ti)
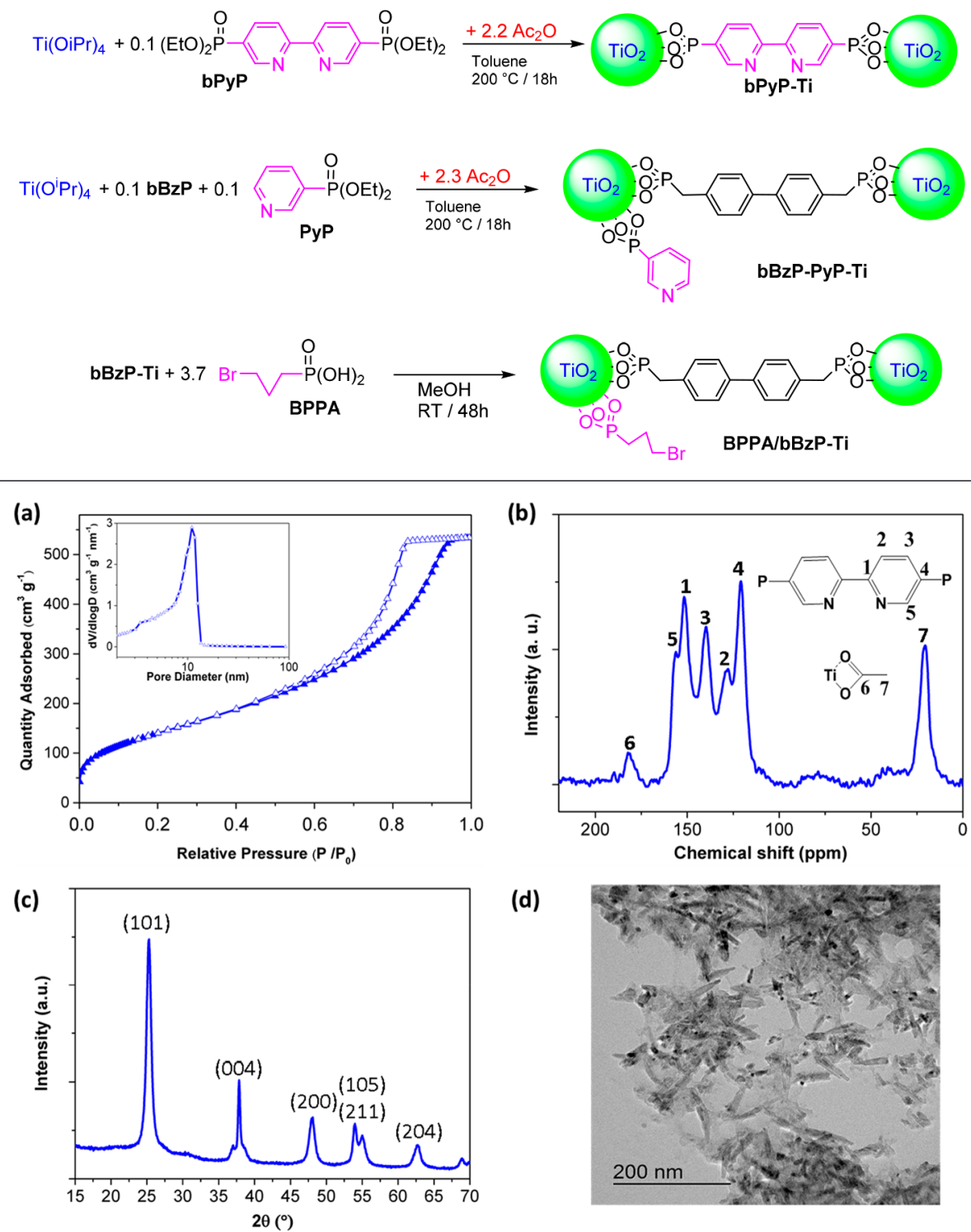

(d)

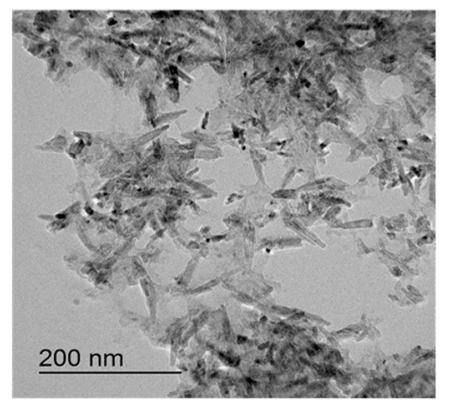

Figure 6. Characterization of bPyP-Ti: (a) $\mathrm{N}_{2}$ adsorption-desorption isotherm at $77 \mathrm{~K}$ and corresponding BJH pore size distribution (inset); (b) ${ }^{13} \mathrm{C}$ solid-state CP-MAS NMR spectrum; (c) powder X-ray diffraction pattern; (d) TEM image.

Table 2. Textural Data for Functional Bridged Titania Bisphosphonate Samples

\begin{tabular}{lcccc}
\multicolumn{1}{c}{ sample } & $S_{\mathrm{BET}}{ }^{a}\left(\mathrm{~m}^{2} \mathrm{~g}^{-1}\right)$ & $V_{\mathrm{p}}{ }^{b}\left(\mathrm{~cm}^{3} \mathrm{~g}^{-1}\right)$ & $V_{\mu}{ }^{c}\left(\mathrm{~cm}^{3} \mathrm{~g}^{-1}\right)$ & $D_{\mathrm{p}}{ }^{d}(\mathrm{~nm})$ \\
bPyP-Ti & 520 & 0.93 & 0.06 & 6.5 \\
bBzP-PyP-Ti & 830 & 1.37 & 0.17 & 9.0 \\
BAPA/bBzP-Ti & 314 & 0.75 & 0.04 & 11.4
\end{tabular}

${ }^{a}$ BET specific surface area. ${ }^{b}$ Total pore volume at $P / P_{0}=0.99$, ${ }^{c}$ Volume of micropores estimated by DFT analysis, ${ }^{d} \mathrm{BJH}$ average pore diameter in the 2 to $80 \mathrm{~nm}$ range calculated from the desorption branch.

${ }^{13} \mathrm{C}$ NMR indicates that the bipyridyl groups are not damaged during the synthesis. As in the case of bBzP-Ti, XRD indicates the formation of anatase nanorods and ${ }^{31} \mathrm{P}$ NMR shows the presence of fully condensed bisphosphonate groups (Figure S9). Electron microscopy images (Figure 6d and Figure S10) show that these nanorods assemble to form a three-dimensional mesoporous network, as in the case of bBzP$\mathrm{Ti}$, demonstrating the generality of our synthetic route.

The adsorption of $\mathrm{Cu}^{2+}$ ions was investigated to determine the adsorption capacity of $\mathrm{bPyP}-\mathrm{Ti}$ and evaluate the accessibility of the bridging bipyridyl groups (see Figure S11 and Table S3). The maximum adsorption capacity was 0.87

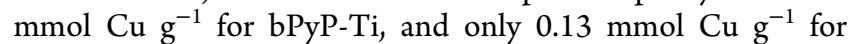
bBzP-Ti. Accordingly, $\mathrm{Cu}^{2+}$ adsorption by bPy-Ti is mostly due to the presence of bipyridyl groups and at least $86 \%$ of these groups are accessible, as calculated from the bipyridyne content $\left(0.86 \mathrm{mmol} \mathrm{g}^{-1}\right.$, see Table S1) after subtraction of the value found for bBzP-Ti, and assuming the formation of a $1: 1$ complex. The maximum $\mathrm{Cu}^{2+}$ adsorption capacity of bPyP-Ti is similar to values reported for diamine-functionalized organosilica sorbents, ${ }^{48}$ such as PMOS cogels, ${ }^{49}$ hexagonal silica $\operatorname{cogels}^{50}$ or postmodified SBA15. ${ }^{51}$ Conversely, the maximum adsorption capacity reported for periodic mesoporous phosphonate hybrid materials prepared from ethyl- 
enediamine tetra(methylene phosphonic acid) and $\mathrm{TiCl}_{4}$ was much lower $(36.5 \mu \mathrm{mol} / \mathrm{g})$, indicating a very low accessibility of the diamine groups in these materials. ${ }^{52}$

Two samples were prepared to illustrate the functionalization with monophosphonate groups. The first one, bBzP-PyP$\mathrm{Ti}$, was obtained by cogelation using 0.1 equiv of both diethylpyridin-3-ylphosphonate and $\mathrm{bBzP}$, while the second sample, BPPA/bBzP-Ti was prepared by surface modification of bBzP-Ti with 3-bromopropyl phosphonic acid (Scheme 2). Functionalization with a bromoalkyl group opens many possibilities for postfunctionalization, notably for the grafting of polymer chains by atom transfer radical polymerization (ATRP).

Both bBzP-PyP-Ti and BPPA/bBzP-Ti samples exhibit a mesoporous texture (Figure $7 \mathrm{a}$ ). ${ }^{31} \mathrm{P}$ MAS NMR (Figure $7 \mathrm{~b}$ )
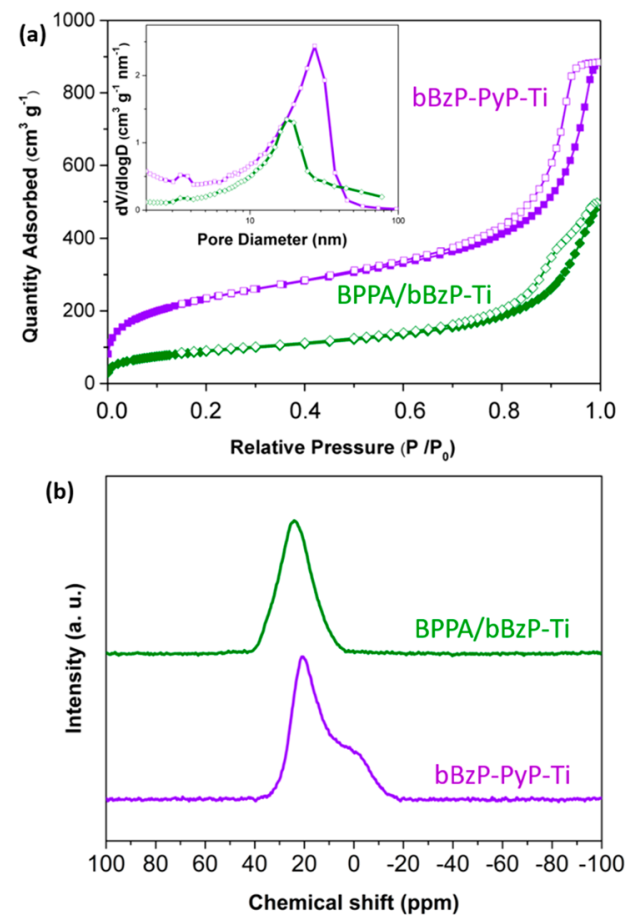

Figure 7. Textural and structural characteristics of bBzP-Py-Ti (purple) and BPPA/bBzP-Ti (olive): (a) $\mathrm{N}_{2}$ adsorption-desorption isotherms at $77 \mathrm{~K}$ and corresponding $\mathrm{BJH}$ pore size distributions (inset); (b) ${ }^{31} \mathrm{P}$ soli-state MAS NMR spectra.

and ${ }^{13} \mathrm{C}$ MAS NMR (Figure S12) confirm the incorporation of the functional groups. The functional group contents are quite high, between 0.80 and $1.03 \mathrm{mmol} \mathrm{g}^{-1}$ (Table S1). The specific surface area $\left(830 \mathrm{~m}^{2} \mathrm{~g}^{-1}\right)$ and pore volume $\left(1.37 \mathrm{~cm}^{3}\right.$ $\mathrm{g}^{-1}$ ) of the Cogel bBzP-Py-Ti are even higher than those of bBzP-Ti. On the other hand, the postmodification of bBzP-Ti leads to a decrease in specific surface area and pore volume (Table 2); however, this method could be useful in the case of thermally sensitive functional groups.

\section{CONCLUSIONS}

In summary, we demonstrate a simple and original nonhydrolytic sol-gel route giving access to a promising family of porous nonsiliceous hybrid materials, bridged titania bisphosphonates. The final materials can be described as titanium oxide nanodomains assembled by rigid, completely condensed bisphosphonate groups. The porosity of these materials can be adjusted over a wide range simply by playing on reaction parameters. Furthermore, functional bridged titania bisphosphonates with high contents of functional groups can be obtained by several methods involving either functional bisphosphonates or easily available monophosphonate esters or monophosphonic acids. The stability over a wide $\mathrm{pH}$ range of these original hybrid materials combined to their tunable texture and functionnality makes them complementary of other mesoporous hybrid materials such as organosilicas or metal phosphonates and opens exciting opportunities notably in the fields of heterogeneous catalysis, drug delivery, ionexchange, or adsorption/separation.

\section{EXPERIMENTAL SECTION}

4.1. Chemicals. Titanium(IV) isopropoxide $\left(\mathrm{Ti}\left(\mathrm{O}^{\mathrm{i}} \mathrm{Pr}\right)_{4}, 97 \%\right)$ and acetic anhydride $\left(\mathrm{Ac}_{2} \mathrm{O}, 99 \%\right)$ were purchased from SigmaAldrich. 4,4'-Bis (diethylphosphonomethyl)biphenyl (bBzP, 98\%) and 3-Bromopropylphosphonic acid (BPPA) were purchased from Tokyo Chemical Industry. Diethylpyridin-3-ylphosphonate (PyP) and tetraethyl 2,2'-bipyridine-5,5'-bisphosphonate ( $\mathrm{bPyP})$ were purchased from SiKEMIA in Montpellier. Copper(II) nitrate hemi(pentahydrate) was purchased from Sigma-Aldrich. All these reactants were used without further purification. Toluene (Sigma-Aldrich, 99.7\%) was dried over a PureSolve MD5 solvent purification system $\left(\mathrm{H}_{2} \mathrm{O}<10\right.$ ppm, controlled with a Karl Fischer coulometer).

4.2. Synthesis. To avoid the presence of water, the addition of reactants and sealing of the autoclaves were carried out in a glovebox under an argon atmosphere ( $<10 \mathrm{ppm}$ of water).

4.2.1. bBzP-Ti. To a solution of titanium(IV) isopropoxide $(1.50 \mathrm{~g}$, $5.25 \mathrm{mmol}$ ) in toluene $(8 \mathrm{~mL}), 4,4^{\prime}$-bis(diethylphosphonomethyl)biphenyl, bBzP, $(0.24 \mathrm{~g}, 0.53 \mathrm{mmol})$ then acetic anhydride $(1.18 \mathrm{~g}$, $11.55 \mathrm{mmol}$ ) were added slowly. After stirring for $5 \mathrm{~min}$, the obtained solution was transferred to a stainless steel autoclave (Parr Instruments) with a PTFE lining $(23 \mathrm{~mL})$, which was then sealed. The autoclave was heated in an oven at $200{ }^{\circ} \mathrm{C}$ for $18 \mathrm{~h}$ under autogenous pressure. After reaction, the resulting monolithic gel was thoroughly washed with ether $(5 \times 30 \mathrm{~mL})$ and acetone $(5 \times 30 \mathrm{~mL})$. The gel was dried under vacuum $(100 \mathrm{~Pa})$ at $120{ }^{\circ} \mathrm{C}$ and finally ground into a fine powder for characterization. \%C 15.9; P/Ti 0.22.

Tuning the Texture of $\mathrm{bBz} P-\mathrm{Ti}$. To tune the texture of the $\mathrm{bBzP}-\mathrm{Ti}$, we have performed additional experiments , changing either the volume of toluene ( 4 and $16 \mathrm{~mL}$ for the synthesis of bBzP-Ti-4 $\mathrm{mL}$ and bBzP-Ti-16 $\mathrm{mL}$, respectively) or the reaction temperature (180 and $220{ }^{\circ} \mathrm{C}$ for the synthesis of bBzP-Ti-180 ${ }^{\circ} \mathrm{C}$ and bBzP-Ti$220{ }^{\circ} \mathrm{C}$, respectively), or the reaction time ( 9 and $36 \mathrm{~h}$ for the synthesis of bBzP-Ti-9h and bBzP-Ti-36h, respectively). All other parameters were kept unchanged.

Functional Hybrid Materials. 4.2.2. bPyP-Ti. This sample was obtained by the same method as bBzP-Ti, but using tetraethyl $2,2^{\prime}$ bipyridine-5,5'-bisphosphonate (bPyP) $(0.23 \mathrm{~g}, 0.53 \mathrm{mmol})$ instead of 4, 4'-bis(diethylphosphonomethyl)biphenyl. \%C 13.6; \%N 2.3; P/ Ti 0.19 .

4.2.3. bBzP-PyP-Ti. To a solution of titanium(IV) isopropoxide $(1.50 \mathrm{~g}, 5.25 \mathrm{mmol})$ in toluene $(8 \mathrm{~mL})$ were slowly added $4,4^{\prime}$ bis(diethylphosphonomethyl)biphenyl, bBzP, $(0.24 \mathrm{~g}, 0.53 \mathrm{mmol}$ ), diethylpyridin-3-ylphosphonate, PyP, $(0.11 \mathrm{~g}, 0.53 \mathrm{mmol})$, and then acetic anhydride $(1.23 \mathrm{~g}, 12.08 \mathrm{mmol})$. After being stirred for $5 \mathrm{~min}$, the obtained solution was transferred to a stainless steel digestion vessel (Parr Instruments) with a PTFE lining $(23 \mathrm{~mL})$. After sealing, the vessel was heated in an oven at $200{ }^{\circ} \mathrm{C}$ for $18 \mathrm{~h}$ under autogenous pressure. After reaction, the resulting monolith was thoroughly washed with ether $(5 \times 30 \mathrm{~mL})$ and acetone $(5 \times 30 \mathrm{~mL})$. It was then dried under reduced pressure $(100 \mathrm{~Pa})$ at $120{ }^{\circ} \mathrm{C}$ and finally ground into a fine powder for characterization, with $\mathrm{P} / \mathrm{Ti} 0.31$.

4.2.4. BPPA/bBzP-Ti. Three hundred milligrams of bBzP-Ti powder $(2.72 \mathrm{mmol})$ was added to a solution of (3-bromopropyl)phosphonic acid, BPPA, $(10 \mathrm{mmol})$ in $\mathrm{MeOH}(100 \mathrm{~mL})$. The mixture was stirred at room temperature for 2 days. After washing 
with $\mathrm{MeOH}(5 \times 30 \mathrm{~mL})$ and acetone $(5 \times 30 \mathrm{~mL})$, the obtained solid was dried under reduced pressure at $120^{\circ} \mathrm{C} . \mathrm{P} / \mathrm{Ti} 0.35 ; \mathrm{Br} / \mathrm{Ti}$ 0.14 .

4.3. Stability Tests. To investigate the hydrolytic stability of bBzP-Ti, we stirred the powder $(0.20 \mathrm{~g})$ for $24 \mathrm{~h}$ at $30^{\circ} \mathrm{C}$ in $400 \mathrm{~mL}$ of aqueous solutions of $\mathrm{HCl}(\mathrm{pHO}$ and $\mathrm{pH} 1)$ or $\mathrm{NaOH}(\mathrm{pH} 12$ and $\mathrm{pH} 13$ ). The hydrothermal stability was tested by refluxing the powder in neutral water at $100{ }^{\circ} \mathrm{C}$ for $2 \mathrm{~h}$. After washing with water and acetone, then drying under reduced pressure at $120{ }^{\circ} \mathrm{C}$, the $\mathrm{N}_{2}$ sorption measurements were repeated.

4.4. Testing of $\mathrm{Cu}^{2+}$ Adsorption by bPyP-Ti and bBzP-Ti. One-hundred eighty milligrams $(1.67 \mathrm{mmol})$ of bPyP-Ti or $185 \mathrm{mg}$ $(1.67 \mathrm{mmol})$ of bBzP-Ti were first washed with water several times to eliminate the acetate groups. They were then separately added to 30 $\mathrm{mL}$ of a copper nitrate standard solution $(10 \mathrm{mM})$. After stirring at room temperature for $24 \mathrm{~h}$, the solid was separated by centrifugation and the $\mathrm{Cu}^{2+}$ content in the supernatant was analyzed by UV-vis spectroscopy. The amount of adsorbed $\mathrm{Cu}^{2+}$ was calculated from the change in $\mathrm{Cu}^{2+}$ concentration of the solutions.

4.5. Characterization. Fourier-transform infrared (FTIR) spectra were collected in ATR mode on a Spectrum II PerkinElmer spectrometer. UV/vis absorption spectra were obtained with a JASCO V-760 UV-visible spectrophotometer in $10 \mathrm{~mm}$ quartz cells (Hellma). Spectra were recorded over the 900-300 nm range with a bandwidth of $1.0 \mathrm{~nm}$ at a rate of $200 \mathrm{~nm} \cdot \mathrm{min}^{-1}$. The powder Xray diffraction (XRD) patterns were collected with a PANalytical X'Pert Pro MPD diffractometer $(\mathrm{CuK} \alpha 1=0.1540598 \mathrm{~nm})$. The scanning electron microscopy (SEM) images were obtained with a Hitachi S-4800 electron microscope. Energy-dispersive X-ray spectroscopy (SEM-EDX) was done on an Oxford Instruments X-Ma ${ }^{\mathrm{N}}$ SDD. The high-resolution transmission electron microscopy (TEM) images were obtained using a JEOL 2200FS-200 kV instrument, and TEM-EDX analyses were acquired with a JEOL CENTURIO detector. Nitrogen adsorption and desorption isotherms were measured at $77 \mathrm{~K}$ with a Micrometrics Triflex apparatus; the specific surface area was determined by the BET method, pore size distribution in the $2-100 \mathrm{~nm}$ range by the $\mathrm{BJH}$ method from the desorption branch, and in the $1-5 \mathrm{~nm}$ range by the DFT method. NMR spectra in solution were recorded using a Bruker AVANCE 400 $\mathrm{MHz}$ spectrometer. Solid state ${ }^{13} \mathrm{C}$ CPMAS NMR spectra were recorded on a VARIAN VNMRS $300 \mathrm{MHz}$ spectrometer using a 3.2 $\mathrm{mm}$ T3 2 channels probe. Rotors were spun at $12 \mathrm{kHz}$. Solid-state ${ }^{31} \mathrm{P}$ magic angle spinning (MAS) NMR experiments were performed on a Varian VNMRS $400 \mathrm{MHz}(9.4 \mathrm{~T})$ spectrometer using a $3.2 \mathrm{~mm}$ Varian T3 HXY MAS probe. Single-pulse experiments were carried out with a spinning rate of $20 \mathrm{kHz}$, a $90^{\circ}$ excitation pulse of $3 \mu \mathrm{s}$, a recycle delay of $30 \mathrm{~s}$ and $100 \mathrm{kHz}$ spinal-64 ${ }^{1} \mathrm{H}$ decoupling. Twohundred transients were recorded. The ${ }^{31} \mathrm{P}$ chemical shift was determined using an external reference, hydroxyapatite $\mathrm{Ca}_{10}\left(\mathrm{PO}_{4}\right)_{6}(\mathrm{OH})_{2}$ at $2.8 \mathrm{ppm}$ (with respect to $\mathrm{H}_{3} \mathrm{PO}_{4}, 85$ wt \% in water).

\section{ASSOCIATED CONTENT}

\section{(s) Supporting Information}

The Supporting Information is available free of charge at https://pubs.acs.org/doi/10.1021/acs.chemmater.9b05095.

Composition of the samples, FTIR spectra, DFT pore size distributions, SEM and TEM images, solution and solid-state NMR spectra, detailed data on stability assessment and on $\mathrm{Cu}^{2+}$ adsorption (PDF)

\section{AUTHOR INFORMATION}

\section{Corresponding Authors}

Johan G. Alauzun - Institut Charles Gerhardt Montpellier, UMR 5253, CNRS-Université de Montpellier-ENSCM, Montpellier Cedex 5 34095, France; (1) orcid.org/0000-00026531-0750; Email: johan.alauzun@umontpellier.fr
P. Hubert Mutin - Institut Charles Gerhardt Montpellier, UMR 5253, CNRS-Universite de Montpellier-ENSCM, Montpellier Cedex 5 34095, France; 이이.org/0000-0002-6031-6467; Email: hubert.mutin@univ-montp2.fr

\section{Author}

Yanhui Wang - Institut Charles Gerhardt Montpellier, UMR 5253, CNRS-Université de Montpellier-ENSCM, Montpellier Cedex 5 34095, France

Complete contact information is available at: https://pubs.acs.org/10.1021/acs.chemmater.9b05095

\section{Author Contributions}

Y.W. performed the experiments. J.A. and P.M. supervised the experiments. P.M. wrote the manuscript with the help of Y.W. and J.A.

\section{Notes}

The authors declare no competing financial interest.

\section{ACKNOWLEDGMENTS}

This work was supported by the European Commission under Grant 720996.

\section{REFERENCES}

(1) Faustini, M.; Nicole, L.; Ruiz-Hitzky, E.; Sanchez, C. History of Organic-Inorganic Hybrid Materials: Prehistory, Art, Science, and Advanced Applications. Adv. Funct. Mater. 2018, 28, 1704158.

(2) Sanchez, C.; Julian, B.; Belleville, P.; Popall, M. Applications of hybrid organic-inorganic nanocomposites. J. Mater. Chem. 2005, 15, 3559-3592.

(3) Judeinstein, P.; Sanchez, C. Hybrid organic-inorganic materials: a land of multidisciplinarity. J. Mater. Chem. 1996, 6, 511-525.

(4) Hoffmann, F.; Cornelius, M.; Morell, J.; Fröba, M. Silica-Based Mesoporous Organic-Inorganic Hybrid Materials. Angew. Chem., Int. Ed. 2006, 45, 3216-3251.

(5) Mizoshita, N.; Tani, T.; Inagaki, S. Syntheses, properties and applications of periodic mesoporous organosilicas prepared from bridged organosilane precursors. Chem. Soc. Rev. 2011, 40, 789-800.

(6) Park, S. S.; Santha Moorthy, M.; Ha, C.-S. Periodic mesoporous organosilicas for advanced applications. NPG Asia Mater. 2014, 6, No. e96.

(7) Urata, C.; Yamada, H.; Wakabayashi, R.; Aoyama, Y.; Hirosawa, S.; Arai, S.; Takeoka, S.; Yamauchi, Y.; Kuroda, K. Aqueous colloidal mesoporous nanoparticles with ethenylene-bridged silsesquioxane frameworks. J. Am. Chem. Soc. 2011, 133, 8102-5.

(8) Copéret, C.; Comas-Vives, A.; Conley, M. P.; Estes, D. P.; Fedorov, A.; Mougel, V.; Nagae, H.; Núnez-Zarur, F.; Zhizhko, P. A. Surface Organometallic and Coordination Chemistry toward SingleSite Heterogeneous Catalysts: Strategies, Methods, Structures, and Activities. Chem. Rev. 2016, 116, 323-421.

(9) Gangu, K. K.; Maddila, S.; Mukkamala, S. B.; Jonnalagadda, S. B. A review on contemporary Metal-Organic Framework materials. Inorg. Chim. Acta 2016, 446, 61-74.

(10) Xuan, W.; Zhu, C.; Liu, Y.; Cui, Y. Mesoporous metal-organic framework materials. Chem. Soc. Rev. 2012, 41, 1677-1695.

(11) Liu, D.; Zou, D.; Zhu, H.; Zhang, J. Mesoporous MetalOrganic Frameworks: Synthetic Strategies and Emerging Applications. Small 2018, 14, 1801454.

(12) Howarth, A. J.; Liu, Y.; Li, P.; Li, Z.; Wang, T. C.; Hupp, J. T.; Farha, O. K. Chemical, thermal and mechanical stabilities of metalorganic frameworks. Nat. Rev. Mater. 2016, 1, 15018.

(13) Gagnon, K. J.; Perry, H. P.; Clearfield, A. Conventional and Unconventional Metal-Organic Frameworks Based on Phosphonate Ligands: MOFs and UMOFs. Chem. Rev. 2012, 112, 1034-1054. 
(14) Zhu, Y.-P.; Ma, T.-Y.; Liu, Y.-L.; Ren, T.-Z.; Yuan, Z.-Y. Metal phosphonate hybrid materials: from densely layered to hierarchically nanoporous structures. Inorg. Chem. Front. 2014, 1, 360-383.

(15) Mutin, P. H.; Guerrero, G.; Vioux, A. Hybrid materials from organophosphorus coupling molecules. J. Mater. Chem. 2005, 15, 3761-3768.

(16) Clearfield, A. Unconventional metal organic frameworks: porous cross-linked phosphonates. Dalton Trans. 2008, 6089-6102.

(17) Dines, M. B.; Cooksey, R. E.; Griffith, P. C.; Lane, R. H. Mixedcomponent layered tetravalent metal phosphonates phosphates as precursors for microporous materials. Inorg. Chem. 1983, 22, 10031004.

(18) Vasylyev, M.; Neumann, R. Preparation, characterization, and catalytic aerobic oxidation by a vanadium phosphonate mesoporous material constructed from a dendritic tetraphosphonate. Chem. Mater. 2006, 18, 2781-2783.

(19) Kimura, T. A New Family of Nonsiliceous Porous Hybrids from Bisphosphonates. J. Nanosci. Nanotechnol. 2013, 13, 2461-2470.

(20) Kimura, T. Synthesis of Novel Mesoporous Aluminum Organophosphonate by Using Organically Bridged Diphosphonic Acid. Chem. Mater. 2003, 15, 3742-3744.

(21) El Haskouri, J.; Guillem, C.; Latorre, J.; Beltran, A.; Beltran, D.; Amoros, P. S+I- Ionic Formation Mechanism to New Mesoporous Aluminum Phosphonates and Diphosphonates. Chem. Mater. 2004, $16,4359-4372$.

(22) Ma, T.-Y.; Zhang, X.-J.; Yuan, Z.-Y. Hierarchical Meso-/ Macroporous Aluminum Phosphonate Hybrid Materials as Multifunctional Adsorbents. J. Phys. Chem. C 2009, 113, 12854-12862.

(23) Ma, T. Y.; Li, H.; Tang, A. N.; Yuan, Z. Y. Ordered, Mesoporous Metal Phosphonate Materials with Microporous Crystalline Walls for Selective Separation Techniques. Small 2011, 7, 1827-1837.

(24) Zhu, Y.-P.; Ren, T.-Z.; Yuan, Z.-Y. Insights into mesoporous metal phosphonate hybrid materials for catalysis. Catal. Sci. Technol. 2015, 5, 4258-4279.

(25) Luca, V.; Tejada, J. J.; Vega, D.; Arrachart, G.; Rey, C. Zirconium(IV)-Benzene Phosphonate Coordination Polymers: Lanthanide and Actinide Extraction and Thermal Properties. Inorg. Chem. 2016, 55, 7928-7943.

(26) Veliscek-Carolan, J.; Rawal, A.; Luca, V.; Hanley, T. L. Zirconium phosphonate sorbents with tunable structure and function. Microporous Mesoporous Mater. 2017, 252, 90-104.

(27) Li, H.; Sun, Y.; Yuan, Z.-Y.; Zhu, Y.-P.; Ma, T.-Y. Titanium Phosphonate Based Metal-Organic Frameworks with Hierarchical Porosity for Enhanced Photocatalytic Hydrogen Evolution. Angew. Chem., Int. Ed. 2018, 57, 3222-3227.

(28) Ciesla, U.; Schüth, F. Ordered mesoporous materials. Microporous Mesoporous Mater. 1999, 27, 131-149.

(29) Fraile, J. M.; Garcia, J. I.; Mayoral, J. A.; Vispe, E.; Brown, D. R.; Naderi, M. Is MCM-41 really advantageous over amorphous silica? The case of grafted titanium epoxidation catalysts. Chem. Commun. 2001, 1510-1511.

(30) Guidotti, M.; Ravasio, N.; Psaro, R.; Ferraris, G.; Moretti, G. Epoxidation on titanium-containing silicates: do structural features really affect the catalytic performance? J. Catal. 2003, 214, 242-250.

(31) Eftekhari, A.; Fan, Z. Ordered mesoporous carbon and its applications for electrochemical energy storage and conversion. Mater. Chem. Frontiers 2017, 1, 1001-1027.

(32) Rolison, D. R. Catalytic Nanoarchitectures-the Importance of Nothing and the Unimportance of Periodicity. Science 2003, 299, 1698.

(33) Guerrero, G.; Mutin, P. H.; Vioux, A. Mixed nonhydrolytic/ hydrolytic sol-gel routes to novel metal oxide/phosphonate hybrids. Chem. Mater. 2000, 12, 1268-1272.

(34) Guerrero, G.; Mutin, P.; Vioux, A. Organically modified aluminas by grafting and sol-gel processes involving phosphonate derivatives. J. Mater. Chem. 2001, 11, 3161-3165.

(35) Maillet, C.; Janvier, P.; Pipelier, M.; Praveen, T.; Andres, Y.; Bujoli, B. Hybrid materials for catalysis? Design of new phosphonate- based supported catalysts for the hydrogenation of ketones under hydrogen pressure. Chem. Mater. 2001, 13, 2879-2884.

(36) Kimura, T. Molecular Design of Bisphosphonates To Adjust Their Reactivity toward Metal Sources for the Surfactant-Assisted Synthesis of Mesoporous Films. Angew. Chem., Int. Ed. 2017, 56, 13459-13463.

(37) Mutin, P. H.; Vioux, A. Nonhydrolytic Processing of OxideBased Materials: Simple Routes to Control Homogeneity, Morphology, and Nanostructure. Chem. Mater. 2009, 21, 582-596.

(38) Wang, Y. H.; Mutin, P. H.; Alauzun, J. G. One-step nonhydrolytic sol-gel synthesis of mesoporous $\mathrm{TiO} 2$ phosphonate hybrid materials. Beilstein J. Nanotechnol. 2019, 10, 356-362.

(39) Wang, Y. H.; Kim, S.; Louvain, N.; Alauzun, J. G.; Mutin, P. H. Acetic Anhydride as an Oxygen Donor in the Non-Hydrolytic Sol-Gel Synthesis of Mesoporous TiO2 with High Electrochemical Lithium Storage Performances. Chem. - Eur. J. 2019, 25, 4767-4774.

(40) Thommes, M.; Kaneko, K.; Neimark, A. V.; Olivier, J. P.; Rodriguez-Reinoso, F.; Rouquerol, J.; Sing, K. S.W. Physisorption of gases, with special reference to the evaluation of surface area and pore size distribution (IUPAC Technical Report). Pure Appl. Chem. 2015, 87, 1051.

(41) Kruk, M.; Jaroniec, M.; Ko, C. H.; Ryoo, R. Characterization of the Porous Structure of SBA-15. Chem. Mater. 2000, 12, 1961-1968.

(42) Wang, S.; Kravchyk, K. V.; Pigeot-Rémy, S.; Tang, W.; Krumeich, F.; Wörle, M.; Bodnarchuk, M. I.; Cassaignon, S.; Durupthy, O.; Zhao, S.; Sanchez, C.; Kovalenko, M. V. Anatase $\mathrm{TiO} 2$ Nanorods as Cathode Materials for Aluminum-Ion Batteries. ACS Applied Nano Materials 2019, 2, 6428-6435.

(43) Miyazaki, S.; Ota, S.; Morisato, K.; Nakanishi, K.; Ohira, M.; Tanaka, N. Preparation and evaluation of monolithic silica columns for HPLC. Chromatography 2011, 32, 87-94.

(44) Ma, T. Y.; Yuan, Z. Y. Functionalized periodic mesoporous titanium phosphonate monoliths with large ion exchange capacity. Chem. Commun. 2010, 46, 2325-2327.

(45) Nijhuis, T. A.; Beers, A. E. W.; Vergunst, T.; Hoek, I.; Kapteijn, F.; Moulijn, J. A. Preparation of monolithic catalysts. Catal. Rev.: Sci. Eng. 2001, 43, 345-380.

(46) Schmidt, J.; Vogelsberger, W. Aqueous Long-Term Solubility of Titania Nanoparticles and Titanium(IV) Hydrolysis in a Sodium Chloride System Studied by Adsorptive Stripping Voltammetry. J. Solution Chem. 2009, 38, 1267-1282.

(47) Clearfield, A., The Early History and Growth of Metal Phosphonate Chemistry. In Metal Phosphonate Chemistry: From Synthesis to Applications; The Royal Society of Chemistry: 2012; Chapter 1, pp 1-44s.

(48) Walcarius, A.; Mercier, L. Mesoporous organosilica adsorbents: nanoengineered materials for removal of organic and inorganic pollutants. J. Mater. Chem. 2010, 20, 4478-4511.

(49) Burleigh, M. C.; Markowitz, M. A.; Spector, M. S.; Gaber, B. P. Amine-Functionalized Periodic Mesoporous Organosilicas. Chem. Mater. 2001, 13, 4760-4766.

(50) Bois, L.; Bonhommé, A.; Ribes, A.; Pais, B.; Raffin, G.; Tessier, F. Functionalized silica for heavy metal ions adsorption. Colloids Surf., A 2003, 221, 221-230.

(51) Aguado, J.; Arsuaga, J. M.; Arencibia, A.; Lindo, M.; Gascón, V. Aqueous heavy metals removal by adsorption on amine-functionalized mesoporous silica. J. Hazard. Mater. 2009, 163, 213-221.

(52) Ma, T.-Y.; Lin, X.-Z.; Yuan, Z.-Y. Periodic mesoporous titanium phosphonate hybrid materials. J. Mater. Chem. 2010, 20, 7406-7415. 